\title{
Paisajes agrarios y transformaciones sociales en el San Juan Mayo y sureste de la cuenca de Pozuelos (Provincia de Jujuy, Argentina)
}

\author{
Valeria Franco Salvi, Carlos Angiorama y Alexis Coronel \\ Recibido 01 de agosto 2018. Aceptado 11 de febrero 2019
}

\begin{abstract}
RESUMEN
En este artículo se presentan los resultados obtenidos del estudio del paisaje agrario de dos áreas arqueológicas ocupadas por poblaciones con rasgos identitarios compartidos a las que, en términos de su cultura material, se las conoce como "cultura Yavi", antecesora directa de la nación Chicha. En el análisis se consideran las transformaciones que se desarrollaron durante los siglos X-XVI d.C., principalmente, el impacto que tuvo la desintegración de las redes de interacción del "Horizonte Medio", los conflictos endémicos del siglo XIII en los valles y la relación con el Tawantinsuyu. Ambos poblados se vieron afectados por las mismas coyunturas, aunque las lógicas sociales para afrontar tales circunstancias no se condicen con las esperadas por el discurso arqueológico tradicional. A partir de un análisis crítico del paisaje agrario fue posible reconocer que los procesos de intensificación agrícola de la cuenca media del río de San Juan fueron gestionados mediante trabajo comunitario, mientras que en el asentamiento de Moreta, el manejo de campos de cultivo a una escala supradoméstica en el área no conllevó a la intensificación de la producción. De esta manera, los datos obtenidos contradicen interpretaciones precedentes que, frente a estos eventos, asumen procesos de intensificación agrícola y desigualdad social como dos factores complementarios.
\end{abstract}

Palabras clave: Paisaje agrario; Transformaciones sociales; Puna de Jujuy.

\begin{abstract}
AGRARIAN LANDSCAPES AND SOCIAL TRANSFORMATIONS IN SAN JUAN MAYO AND SOUTHEAST OF THE POZUELOS BASIN, PROVINCE OF JUJUY, ARGENTINE. This article presents the results obtained from the study of the agrarian landscape of two archaeological areas occupied by populations with shared identity traits that, in terms of their material culture, are known as the Yavi culture, direct ancestors of the Chicha nation. The analysis concerned the transformations that took place during the tenth through sixteenth centuries $\mathrm{AD}$, principally the impact of the disintegration of the exchange networks of the "middle horizon," endemic conflict, and the formation of Tawantinsuyu. Both towns studied were affected by the same set of circumstances, although the social logic used to address events contradicts traditional archaeological discourse. Based on a critical analysis of the agrarian landscape, it was possible to recognize that the processes of agricultural intensification in the middle basin of the San Juan river were managed through community work. In contrast, the supra-domestic management of agricultural fields in the settlement of Moreta did not lead to the intensification of production. These examples contradict previous explanations that, when faced with such events, assume that processes of agricultural intensification and social inequality are complementary factors.
\end{abstract}

Keywords: Agrarian landscape; Social transformations; Jujuy puna.

Valeria Franco Salvi. Consejo Superior de Investigaciones Científicas y Técnicas (CONICET). Instituto de Humanidades (IDH). Pab. Agustín Tosco, primer piso, Ciudad Universitaria, Córdoba. E-mail: valefrancosalvi@unc.edu.ar

Carlos Angiorama. CONICET. Instituto Superior de Estudios Sociales (ISES). San Lorenzo 429. Tucumán. E-mail: carlosangiorama@gmail.com

Alexis Coronel. CONICET. ISES. San Lorenzo 429. Tucumán. E-mail: alenell2@hotmail.com 


\section{INTRODUCCIÓN}

En el presente trabajo se estudia la trayectoria histórica del paisaje agrario en un proceso de larga duración -siglos X-XVI d.C.- a partir del análisis de la microrregión "El Angosto", en la cuenca media del río Grande de San Juan Mayo y el asentamiento de Moreta, al sur de la cuenca de Pozuelos, en la provincia de Jujuy, República Argentina. Estas áreas se encuentran a 40 kilómetros de distancia entre sí y presentan dos fajas de altitud diferentes, lo cual constituye un punto de comparación interesante a los fines de dilucidar cómo se cultivaba en ellas y cómo se constituían en una misma entidad sociocultural -chichas y sus antecesores- en paisajes naturales disímiles (Figura 1).

Los estudios referidos a los sectores de cultivo en el Noroeste argentino (NOA) se han focalizado, durante las décadas de 1980 y 1990, en la observación -i.e., cálculo de la extensión de las áreas de cultivo- y, por consiguiente, deducción del nivel de complejidad tecnológica y social (i.e., agricultura intensiva gestionada por determinados personajes, o extensiva regulada por unidades domésticas) (Raffino 1988; Albeck 1993; Olivera 2001). De acuerdo con lo que se observaba en la superficie se infería un grado de gestión, y por ende, un periodo particular y una estructura política. En este sentido, numerosas áreas con infraestructura de gran escala se concibieron de forma sincrónica, sin cuestionarse su posible diacronía y contexto social (Quesada 2006).

La arqueología local articula en sus reflexiones la información inferida sobre el uso y la función de los espacios de producción agrícola mediante un relato basado en tipologías y grados de complejidad de carácter lineal (Franco Salvi 2012). En general, en la literatura arqueológica aparece como implícita y aceptada la idea de que algunos tipos de estructuras de cultivo (e.g., canchones) corresponden a sociedades de tipo "simple" del periodo Formativo, mientras que un tipo de construcción de mayor envergadura (e.g., terrazas y andenes) y con sistemas de irrigación (e.g., canales) se relaciona con sociedades "complejas", gestionadas en el marco de un contexto social desigual (Raffino 1988; Albeck 1993, Olivera 2001; Figueroa 2008). En esta línea analítica se deduce que la presencia

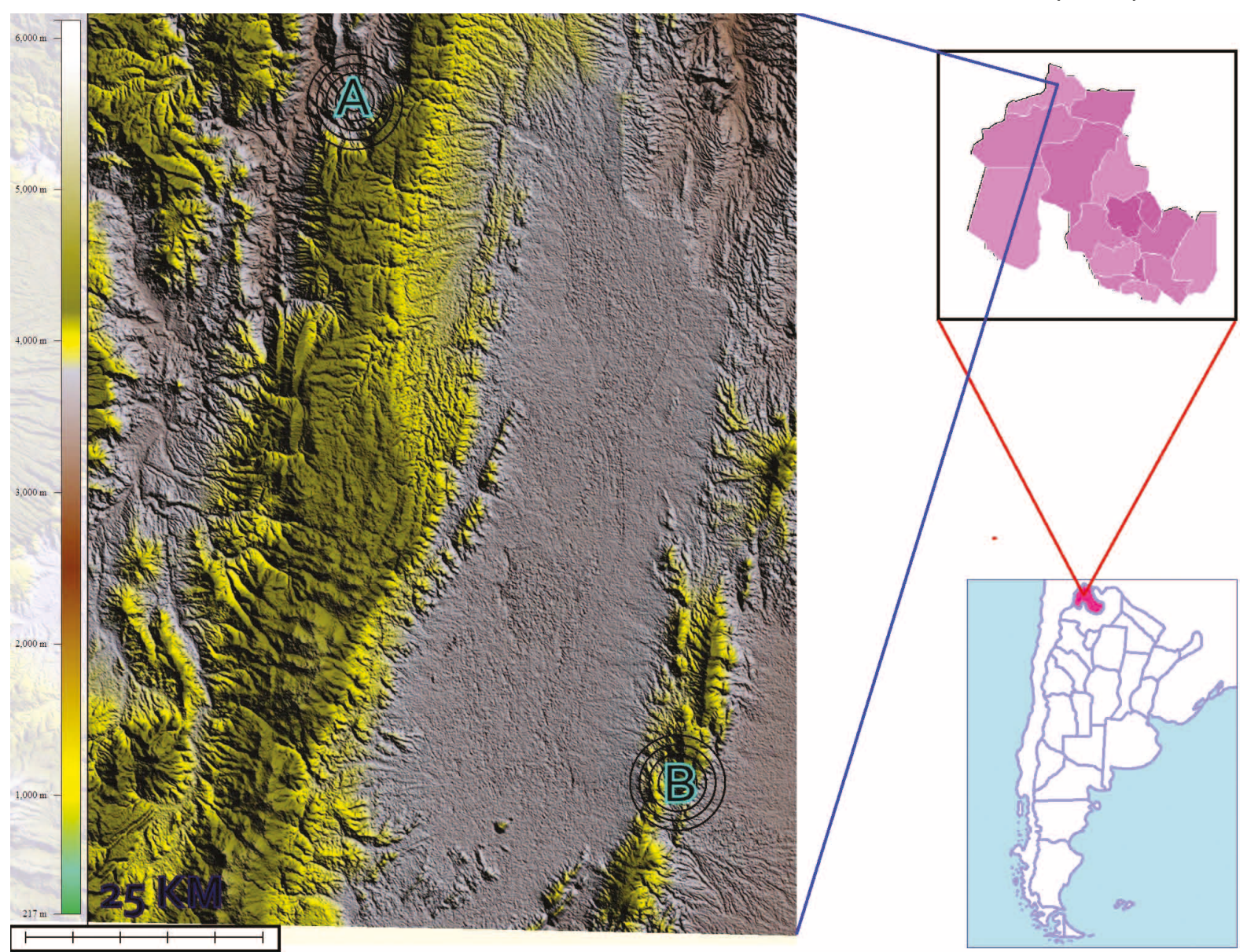

Figura 1. Área de estudio, Puna de Jujuy, Argentina. A) Microrregión "El Angosto" en la cuenca media del río Grande de San Juan Mayo. B) Sector Sur de la cuenca de Pozuelos. 
de campos de cultivo en áreas segregadas del sector residencial se corresponde con la existencia de organizaciones con poder centralizado, jerarquías sociales y así sucesivamente.

Para el NOA, algunos autores han propuesto que entre los siglos X-XVI d.C. se gestaron formas agrícolas de explotación más intensiva, orientadas a la generación de excedentes y organizadas a partir de un sistema de jerarquías sociales (Raffino 1988; Albeck 1993; Sempé 2005, entre otros). Sin embargo, nuevas investigaciones sostienen que, más que desigualdades sociales institucionalizadas, se presentaron otras formas sociales basadas en el trabajo comunitario de integración laxa y sin estructuras jerárquicas (Nielsen 2006; Acuto 2007), lo que significa que la constitución del paisaje agrario se enmarca en una red de relaciones diferente $y$, por ende, la fórmula agricultura intensiva = desigualdad social y agricultura extensiva = igualdad social debe ser discutida y contrastada.

Puesto que los cambios que se observan en la manera de producir podrían referirse a numerosos factores y relaciones con un escenario social diverso, nos preguntamos: ¿la intensificación agrícola requiere de una gestión centralizada y con desigualdades institucionalizadas?; ¿la monumentalidad agrícola implica intensificación de la producción? Proponemos buscar las respuestas en dos sectores que fueron ocupados por poblaciones con rasgos identitarios compartidos, esto es, la cuenca media del río Grande de San Juan (en adelante, RGSJM), en la microrregión El Angosto, departamento de Santa Catalina (Nielsen et al. 2015) y la porción sur de la cuenca de Pozuelos en la puna de Jujuy, específicamente los sectores agrícolas Moreta 1 y Carahuasi, cercanos al asentamiento de Moreta (Angiorama et al. 2017).

Cabe señalar que temáticas similares se han discutido en la puna meridional argentina, cuando se analizaron las tecnologías agrícolas y sus implicancias sociales, políticas y económicas en las sociedades prehispánicas, a partir de lo cual se propusieron una serie de revisiones con nuevas vías interpretativas (Quesada 2006; Díaz 2009). Quesada (2006) advierte que la jerarquización social no siempre es consecuencia de la expansión agrícola y que los sistemas de irrigación altamente complejos han convivido muchas veces con un control descentralizado del poder, y presenta un caso concreto en el área de Antofalla. Otro ejemplo lo brinda Díaz (2009) para el sitio "Piedras Negras", donde una notable extensión de cultivo no implicó escalas sociales supradomésticas (Quesada 2006). Estos autores han remarcado semejanzas entre el paisaje de Tebenquiche y Antofalla con el de Morro Relincho, La Mesada, El Alto El Bolsón (Quesada y Korstanje 2010) y Aldea Piedra Negra (Díaz 2009). A diferencia de otros sectores del NOA, esa forma de estructuración espacial permaneció vigente luego del Formativo tanto en el salar de Antofalla (Quesada 2006), como en el valle de El Bolsón (Quesada y Korstanje 2010), por lo cual también llevó a suponer que en algunos ámbitos, en particular en la puna y los valles altos del oeste catamarqueño, la estructuración de la vida campesina en torno a su economía de autosuficiencia pudo haber perdurado en el tiempo (Quesada y Korstanje 2010). En este sentido, podemos observar que estas preguntas exceden la escala local y se hacen ya a un nivel regional, y permiten deconstruir muchas ideas arraigadas provenientes, sobre todo, de perspectivas neoevolucionistas que persisten de diferentes maneras, particularmente, de forma implícita en la literatura arqueológica, pese a las críticas y nuevas perspectivas.

Nuestras tareas arqueológicas se iniciaron mediante teledetección, lo que implicó, en principio, el empleo de programas aplicados para el manejo cuantitativo y cualitativo de variables espaciales. Se integró un paquete de software de sistema de información geográfica -Global Mapper- que nos permitió determinar cuencas hidrográficas para conocer la disponibilidad de agua en los sitios. También empleamos la herramienta View Shed Tool para generar una cuenca visual y así poder establecer la visibilidad de los andenes en Carahuasi. Se consideraron variables topográficas, acceso a recursos, cuencas hídricas y perfiles de elevación de las áreas cultivadas a los fines de generar estrategias de relevamiento para dar cobertura a los distintos espacios que conforman el área de estudio. Una vez finalizada dicha tarea, avanzamos en la identificación, registro y relevamiento de ocupaciones arqueológicas en superficie mediante estación total y fotogrametría. De esta forma se optó por la fotogrametría aérea mediante Drone (Dji Phantom 3 Professional) y se empleó la aplicación PIX4D, a fin de obtener un modelo acumulativo tridimensional.

El plano tridimensional generado nos permitió diferenciar estructuras para el manejo del agua, diseños arquitectónicos, áreas de despedre y numerosas características que presentaban a nivel superficial las áreas de cultivo. Se llevaron a cabo 
prospecciones pedestres, recolecciones superficiales sistemáticas y excavaciones arqueológicas en áreas seleccionadas, y fueron extraídas muestras de suelo a nivel vertical y horizontal. Para situar y fechar cada contexto se utilizó una metodología de registro estratigráfico sobre la base de matrices de Harris (1991) aplicada por Carandini (1997) para la interpretación de la secuencia de construcción de estructuras arquitectónicas. El planteo de Quesada y Maloberti (2012), que trata de una secuenciación de las relaciones de adosamiento de estructuras de cultivo, fue tomado como guía para el análisis de la manera en que fueron agregándose muros y recintos al paisaje, aunque hasta el momento contamos solo con una primera aproximación. Asimismo, estudiamos las características tecnológicas y tipológicas y los cambios morfológicos en las estructuras identificadas con base en los trabajos de Albeck (1993) y los sistemas de canales a partir de la propuesta de Quesada (2006).

\section{LOS CASOS DE ESTUDIO}

\section{Agricultura en las quebradas: sitios Saratarkuna y San Lorenzo 1}

La microrregión denominada "El Angosto" está emplazada entre 3300 y 5500 msnm en la cuenca media del RGSJM. Las precipitaciones promedian los $300 \mathrm{~mm}$ al año y se concentran entre los meses de diciembre y marzo. Durante ese periodo, la caída de granizo es un problema para los agricultores locales, mientras que en los meses secos (abril y septiembre), las heladas afectan notablemente las prácticas productivas.

Nielsen et al. (2015) afirman que durante los siglos XIII-XVI d.C. habría residido en el área una densa población, a juzgar por la cantidad de sitios detectados, ${ }^{1}$ y que serían los antecesores directos de la nación Chicha, aludida en los documentos históricos (Krapovickas 1983).

Los trabajos se efectuaron en dos quebradas, denominadas San Lorenzo 1 y Saratarkuna. Estas áreas fueron elegidas para la práctica de la agricultura por ser abrigadas y por su topografía apta para la irrigación mediante canales. Al tratarse de topografías sumamente accidentadas, se denota una distribución fragmentaria de las áreas de cultivo, a diferencia de las grandes extensiones continuas que se observan en Quebrada de Humahuaca (Albeck 1993). En ambos sectores se realizaron prospecciones y recolecciones superficiales sistemáticas que permitieron identificar un gran porcentaje de alfarería del grupo Yavi/Chicha y unos muy pocos fragmentos cerámicos con estilos foráneos aislados, que posiblemente fueran adquiridos por redes de intercambio con áreas cercanas (Nielsen et al. 2015).

Se identificaron diversos sitios agrícolas, principalmente en los fondos de valle y quebradas con fuentes de agua, que comprenden seis tipos de rasgos en diversas combinaciones: 1) despedres, 2) terrazas, 3) canales, 4) represas, 5) melgas y 6) refugios (Figura 2). Las quebradas relevadas exhiben una recurrente presencia de estructuras agrícolas asociadas, esto es, canal-melga-despedre-andén (Figuras 2 y 3), y se registró que los espacios de producción se corresponden con el diseño irrigado.

En estas quebradas se identificó un sistema de riego centrípeto (Quesada 2006) que toma el agua de los cauces naturales semipermanentes (Qda. de Saratarkuna), y otro por colectores (Nielsen et al. 2015) que aprovechan el agua de Iluvia durante el período estival (Qda. de San Lorenzo). El sistema por colectores consta de varias represas ubicadas contra una gran pared de arenisca que se extiende por kilómetros en el área, que fueron construidas con el fin de almacenar y distribuir el agua de Iluvia. Para potenciar este aporte, se han colocado, en distintos puntos del afloramiento, muros bajos a modo de trabas o colectores que encauzan el escurrimiento hacia las represas. El sistema de riego centrípeto (Quesada 2006) detectado en Saratarkuna se caracteriza por la presencia de un canal principal que se dirige de manera paralela al cauce natural. La toma de agua se realiza en un sector de mayor altitud del río principal o en uno de sus brazos, y desde allí se distribuye, para regresar al acuífero nuevamente. Quesada (2006) sostiene que esta tipología es recurrente en el interior de las quebradas, y sobre todo en secciones de pendientes pronunciadas en sentido transversal a los arroyos.

Otra tipología de canal detectada presenta una pared lateral, y se la puede encontrar en sectores con pendiente. El dispositivo capta el agua de los cauces y la traslada en altura sorteando los obstáculos topográficos e integrando áreas cultivables (principalmente andenes) a una misma red de riego. Para esto, se requirió de un muro contenedor de entre 0,30 a 0,50 m de altura (esta tipología se registró en los cañadones de río). En este contexto, se hallaron también canales con dos paredes laterales que se disponen en el sentido de la pendiente, 

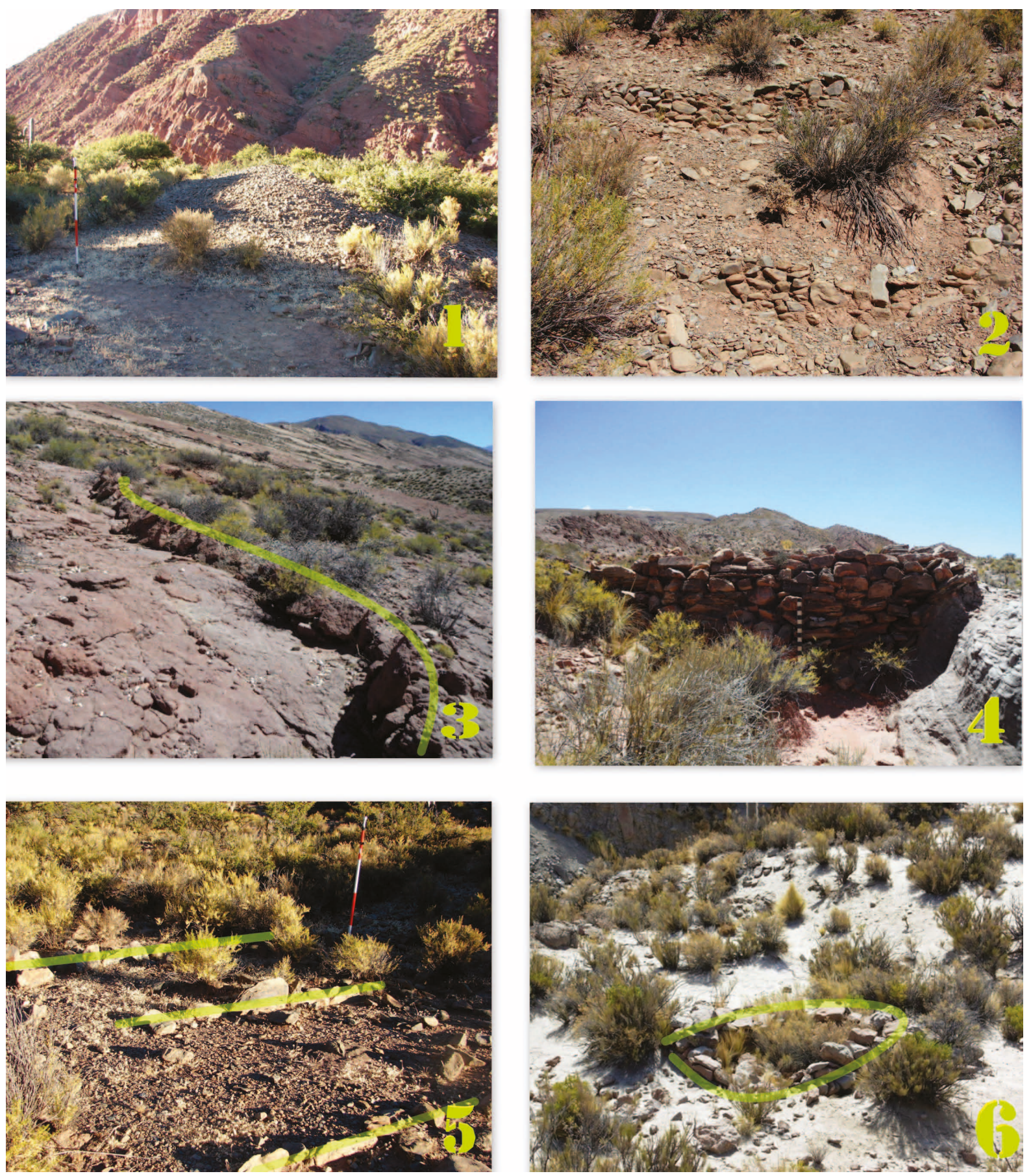

Figura 2. Sitios Saratarkuna y San Lorenzo 1:1) despedres, 2) terrazas, 3) canales, 4) represas, 5) melgas y 6) refugios.

cuya construcción consta de lajas clavadas, aunque también se diferenciaron bloques de mayor solidez. En la mayoría de los casos, no presentan una gran longitud, dado que constituyen cortos segmentos intermedios que se desprenden de manera perpendicular de un canal principal y continúan luego sin solución de continuidad como canales sin pared.

Asociadas a estos sistemas de canales y en sectores de menor pendiente, se registraron numerosas estructuras de piedra que, por sus características, se denominan melgas (Díaz 2009). Estas parcelas se emplazan en el paisaje a modo de cuadrantes definidos por muros simples de piedra, a veces formando cuadrados mayores delimitados por muros más sólidos (dobles), todos repetidamente situados en terrazas fluviales bajas. Las excavaciones estratigráficas nos permitieron advertir que los muros simples observados en superficie poseen varias hiladas bajo tierra, lo que muestra que se trata de una técnica de manejo de los suelos en profundidad (Figura 3). Díaz (2009) las define como "una técnica de inundación donde la superficie agrícola es dividida 

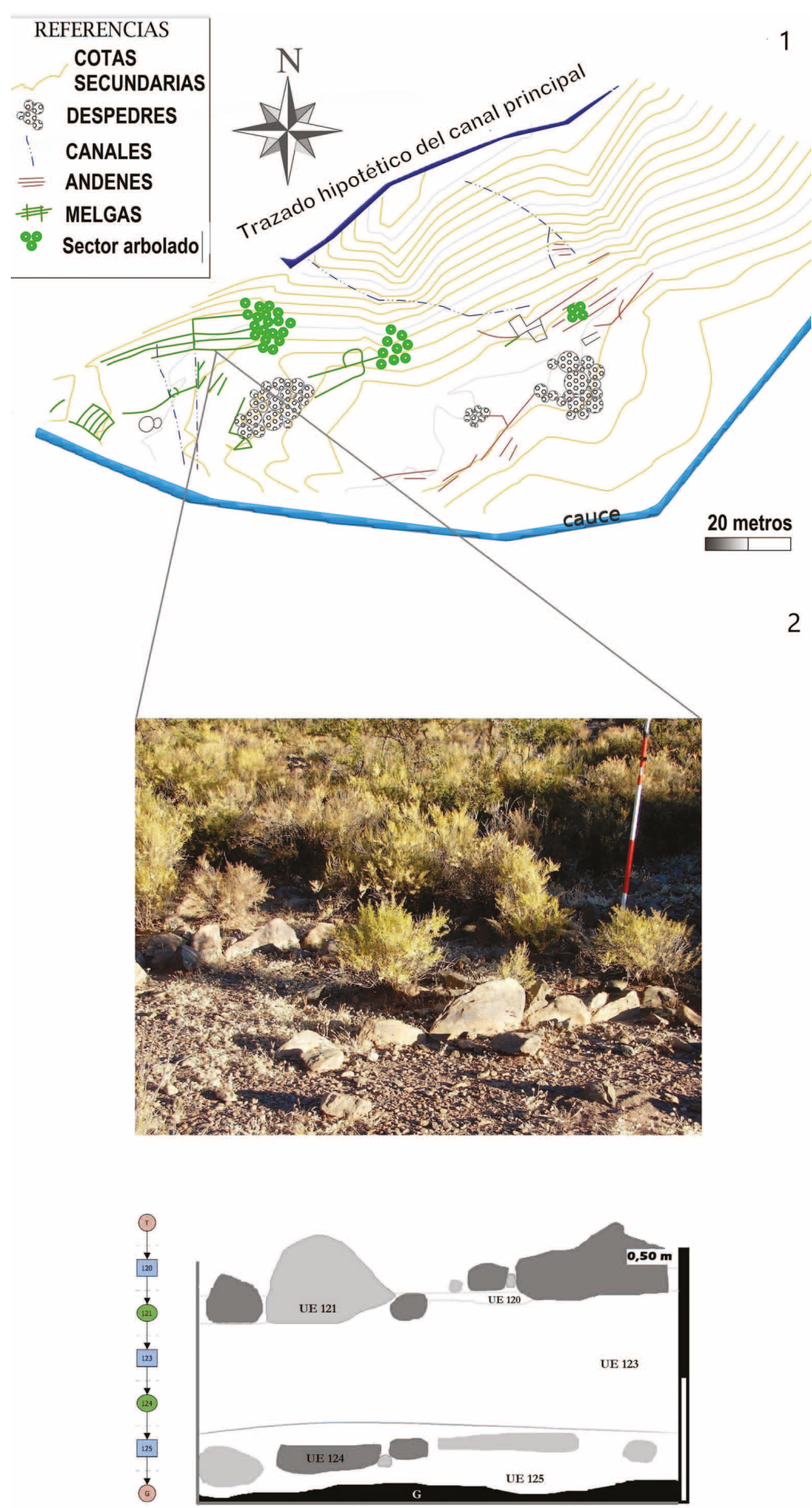

Figura 3. 1) Quebrada de Saratarkuna. Plano de planta de un tramo del sector de cultivo. 2) Sección superior: fotografía de la melga vista desde la superficie (Quebrada de Saratarkuna). Sección inferior: perfil norte y matriz de Harris donde se puede apreciar que poseen varias hiladas bajo tierra.

en pequeños espacios nivelados encontrándose la circulación del agua altamente sistematizada" (Díaz 2009: 53)

Entre las melgas y canales hemos registrado grandes montículos de despedre -producto de la limpieza de las superficies cultivadas- posicionados de forma elongada en el sentido de la pendiente cada 30 metros de distancia entre sí. A modo de conjetura, Albeck (2010) sostiene que, a partir del tamaño del cascajo (i.e., roca) que compone las acumulaciones de despedre, es posible inferir una cronología aproximada; por ejemplo, en momentos inkas, la modalidad del despedrado se realizaba a partir de un rastrillado muy fino del terreno que generaba cascajos pequeños de piedras menudas o grava. El caso de aqueIlos sectores donde el cascajo es de tamaño considerable puede ser relacionado con etapas previas de cultivo, anteriores a los momentos inkaicos (Albeck 2010), situación sostenida a modo de hipótesis por Williams et al. (2010) para la localidad de Gualfín en el Valle Calchaquí. En este caso particular, el nivel de despedrado presenta principalmente bloques con un tamaño promedio de 10-15 $\mathrm{cm}$ de diámetro, lo que nos lleva a inferir como idea preliminar que estos despedres corresponden a momentos preinkaicos.

Los andenes se presentan como superficies niveladas angostas (1-2 $\mathrm{m}$ en el sentido de la pendiente) en agrupaciones que no superan las 10 hileras, sostenidas por muros simples de hasta $1 \mathrm{~m}$ de altura, generalmente ubicados en pendientes pronunciadas, como las laderas de las quebradas. Las melgas y andenes se asocian formando áreas de cultivo, y se extienden desde las terrazas fluviales bajas (melgas) hasta las laderas de las quebradas (andenes).

Las estructuras presentaban un diseño centrado en optimizar el uso del agua y en extender las superficies cultivables. Se trata de un desarrollo agrícola extenso y aislado espacialmente de otras actividades. Los análisis de las muestras de sedimento 
extraídas de estructuras agrícolas han generado hasta ahora cantidades mínimas o nulas de sílicofitolitos debido a factores tafonómicos relacionados con la alcalinidad del suelo. En ese sentido, la única información obtenida sobre las especies potencialmente cultivadas fue extraída de macrorrestos carbonizados de quinoa (Chenopodium quinoa var. quinoa y var. melanospermum), maíz (Zea mays), poroto (Phaseolus vulgaris) y amaranto (Amaranthus caudatus) obtenidos por flotación de excavaciones realizadas en unidades residenciales que serían contemporáneas a dichos campos (Nielsen et al. 2015).

En definitiva, las quebradas presentan una serie de construcciones recurrentes, esto es, la secuencia que incluye canales, melgas, despedres y andenes. A excepción de los andenes, creemos que la secuencia canalmelga-despedre es interdependiente, lo que a su vez implica simultaneidad temporal. ${ }^{2}$ La presencia de melgas involucra canales y despedres como un dispositivo que, en conjunto, funciona para cultivar en estas quebradas. En referencia al abandono de las estructuras, no se observan derrumbes o destrucciones intencionales; tampoco se reconocieron prácticas rituales y/o algún tipo de fenómeno natural que haya incidido en su correcta utilización. Nielsen et al. (2015) sostienen que la zona se habría abandonado cuando el Inka decidió trasladar las poblaciones a otros sectores para prestar servicios al Tawantinsuyu.

\section{Agricultura en las estribaciones: Moreta 1 y Carahuasi}

Desde hace más de una década se vienen desarrollando investigaciones arqueológicas sistemáticas en el sur de Pozuelos (Angiorama et al. 2017). La organización espacial de los asentamientos del período prehispánico tardío se podría definir como "dispersa": espacios rurales conformados por viviendas diseminadas y asociadas a estructuras de cultivo y pastoreo de pequeña escala (i.e. sitios Tabladitas 1, Casa Colorada 2, etc.), siendo muy escasos los conglomerados residenciales para el periodo tardío en la zona (Angiorama et al. 2017). Al parecer, los conflictos endémicos del siglo XIII y la intervención del Estado inka en la vida social local fue contundente en lo relacionado con la organización y significación del espacio.

La cuenca de Pozuelos, emplazada entre los 3500 y 4200 msnm, es parte del extremo meridional del altiplano andino. Las prospecciones realizadas en este sector han permitido identificar un asentamiento arqueológico interpretado como poblado y tambo de Moreta (Angiorama et al. 2017) y dos áreas de cultivo próximas denominadas Moreta 1 y Carahuasi (Figura 4).

El asentamiento Moreta emplazado a $3850 \mathrm{msnm}$ está constituido por un recinto perimetral compuesto (RPC) próximo a un conjunto de estructuras
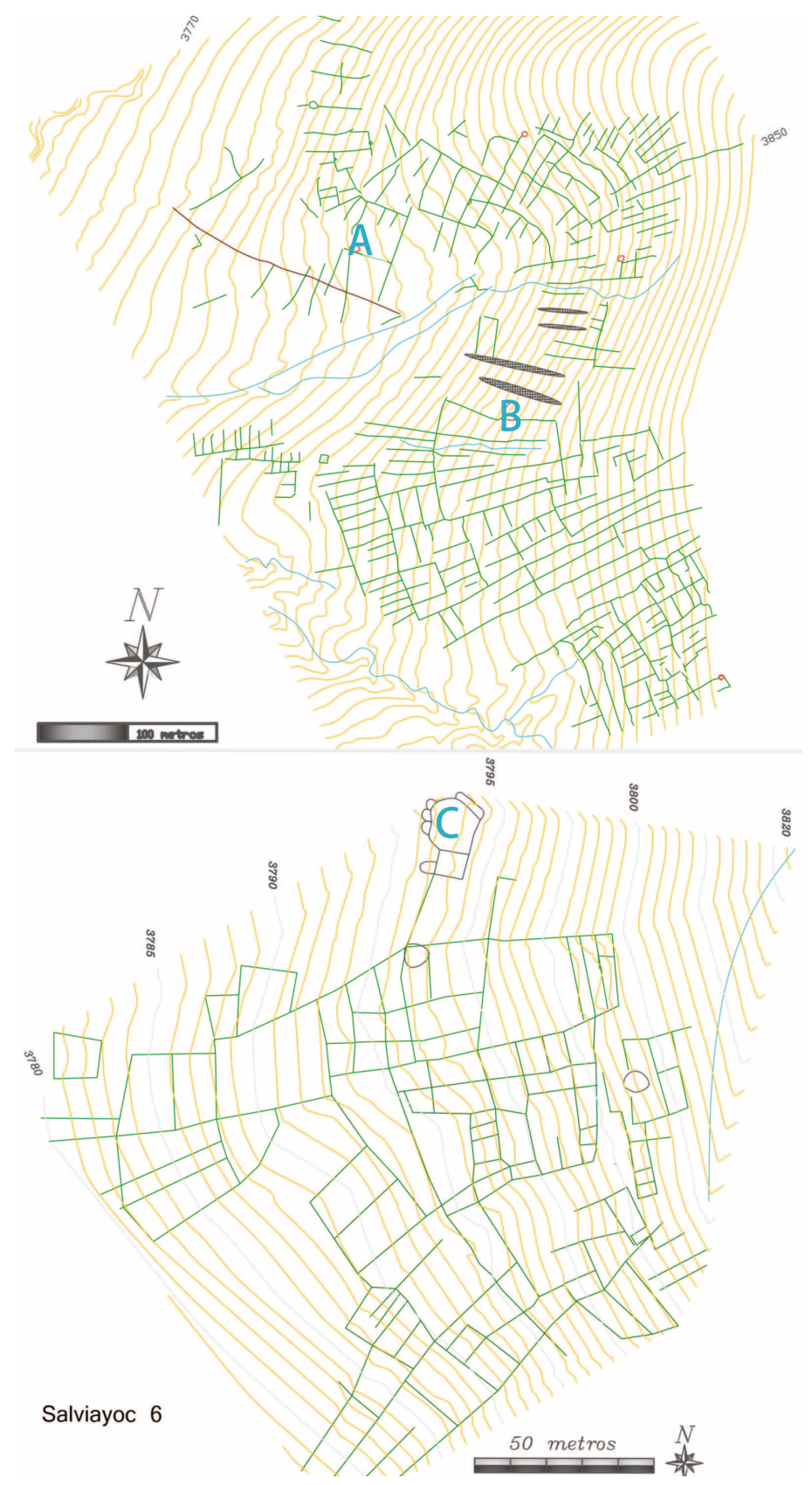

Figura 4. Sectores de cultivo: Arriba: Carahuasi. Abajo: Moreta 1. A: recinto "a". B: recinto "b". C: Estructura residencial "refugio". 
residenciales que cubren un área de unas dos hectáreas. Las estructuras residenciales presentan forma cuadrangular $y / 0$ rectangular y fueron construidas alrededor de un patio central, formando un patrón similar al registrado en otros lugares ocupados por las poblaciones "chicha" entre los siglos XI y XVI. Asimismo, a unos pocos metros en dirección sudeste, en la cima de una colina, se detectó un sector defensivo con estructuras de vivienda y muros de contención (Angiorama et al. 2017). Desde allí se puede observar el Pukara de Rinconada y otros puntos importantes de la región, como la laguna de Pozuelos, el abra de Queta, los andenes de Carahuasi, la cordillera de Lípez, el volcán Granada, etcétera.

En las estribaciones próximas al asentamiento de Moreta se identificaron unas 200 hectáreas de diversas estructuras agrícolas asociadas a fuentes de agua, que comprenden ocho tipos de rasgos en diversas combinaciones: 1) melgas, 2) andenes, 3) terrazas, 4) despedres, 5) grandes líneas transversales, 6) refugios, 7) canales y 8) canchones (Figura 5).

Hasta el momento, en el área de Carahuasi hemos identificado algunos tramos de canales sin pared en las zonas superiores de los espacios con andenería de mayor desnivel, aunque no hallamos el punto de toma (Albeck 1993) desde donde identificar de qué curso y/o vertiente se obtenía el agua para riego. En el sector Moreta 1, además del canal, se habría empleado un sistema de riego por colector para aprovechar el agua de lluvia durante el periodo estival. En los sectores de menor pendiente del sitio se registraron grandes líneas de muros de piedra transversales al curso de agua principal que se habrían regado mediante inundación durante las crecidas para aprovechar la proximidad al cauce. Resulta válido aclarar que los procesos de remoción y erosión impiden identificar la presencia de otras formas de riego en estos sectores.
Moreta 1 se presenta como un conjunto arquitectónico agrícola de construcciones delimitadas por paredes de piedra que forman terrazas, grandes líneas transversales y estructuras circulares aisladas o "refugios". Hasta el momento, se identificó un solo rasgo en el terreno que correspondería a una estructura residencial. Las dataciones radiocarbónicas sobre carbones obtenidos de excavaciones estratigráficas realizadas en uno de los recintos, al que denominamos "refugio", emplazado entre los sectores de cultivo, indican
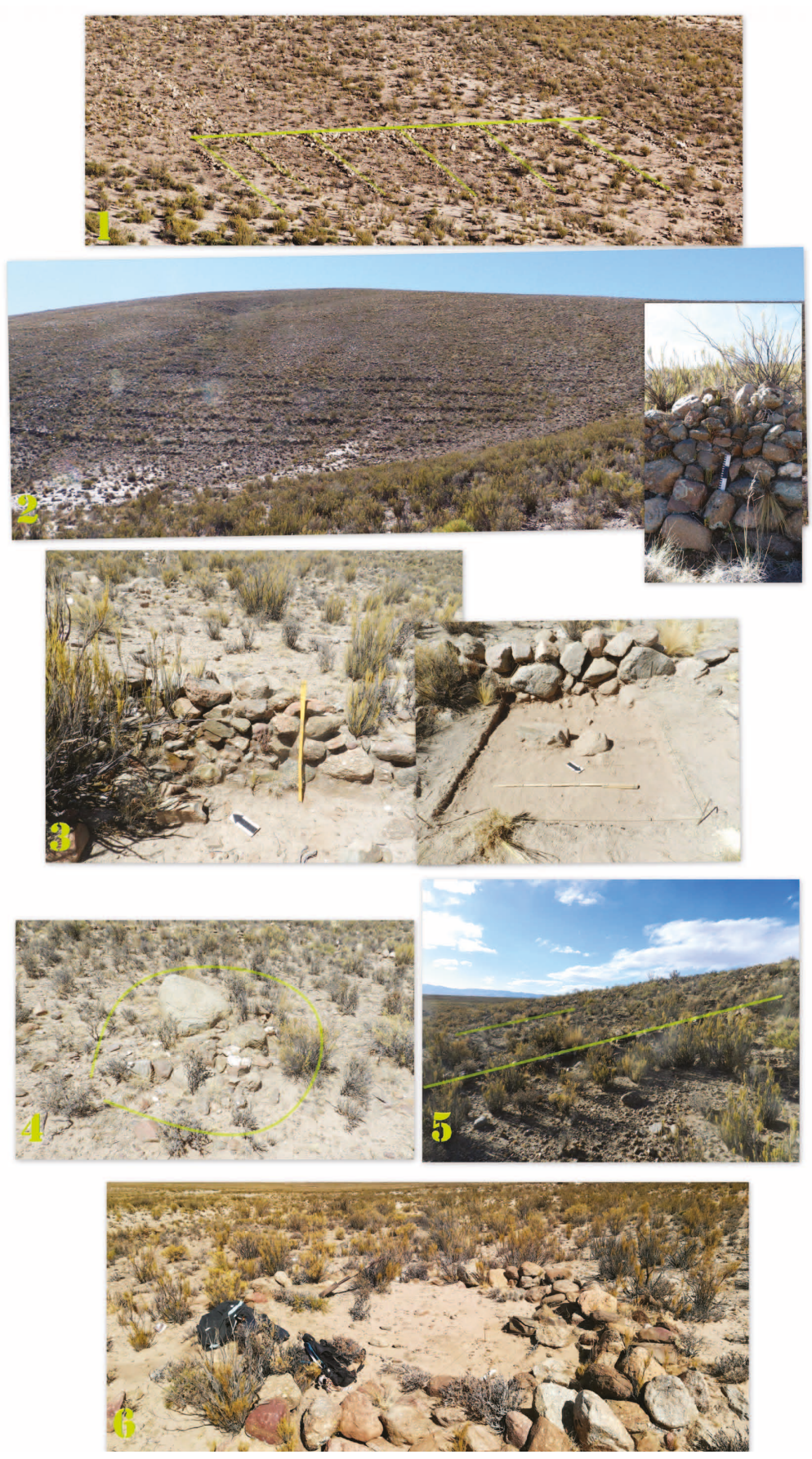

Figura 5. Espacios de cultivo registrados en las proximidades del asentamiento de Moreta. 1) melgas, 2) andenes, 3) terrazas, 4) despedres, 5) grandes líneas transversales y 6) refugios. 
que entre los siglos XIII y XV $\mathrm{XV}^{3}$ probablemente se cultivara en estos sectores.

El sitio "Carahuasi" (Figura 5) reúne una gran variabilidad de estructuras de cultivo; en el paisaje se destacan muros de piedra dispuestos de forma longitudinal y transversal a la pendiente, formando líneas de hasta 200 metros que se extienden desde lo más alto de la quebrada y descienden por la ladera hasta los sectores bajos, traspasando el cauce principal. A medida que aumenta el desnivel (supera un $10 \%$ ), también lo hace la altura de los muros que forman el conjunto agrícola (Figura 4 y 5). En este sentido, las estructuras de piedra van moldeándose de acuerdo con las características del terreno (cambios de pendiente, presencia de cárcavas, etc.) y en relación con las posibilidades de aprovechar la circulación de agua, tanto de lluvia como de cauces.

Un morfotipo recurrente que se reconoce en la región desde las primeras ocupaciones aldeanas hasta el siglo XV d.C. son los canchones y pequeños muros de contención asociados a las viviendas $y$, por ende, de gestión doméstica. Otros tipos de estructuras identificadas y que aparecen entre los siglos X-XV d.C. son las melgas, terrazas y grandes líneas transversales emplazadas sin asociación a viviendas. De acuerdo con la evidencia reunida, podrían haber sido manejadas a escala colectiva y coordinadas por grupos de familias que cohabitaban el poblado chicha, emplazado a 3 kilómetros de distancia. ${ }^{4}$ Por último, conjeturamos la incorporación tardía de un conjunto de andenes que habrían sido gestionados por el Inka a mediados del siglo XV.

En las terrazas fluviales bajas se registraron cientos de melgas definidas como muros simples de piedra, que delimitan cuadrantes situados en terrenos nivelados (Figura 5). Las pequeñas parcelas que se forman permiten una circulación sistemática del agua. En este sitio es posible observar, desde un mismo punto geográfico, tanto las melgas, en los terrenos bajos, como los andenes, en lo más alto de la serranía. Sin embargo, los datos cronológicos absolutos y relativos podrían diferir en antigüedad; por un lado, las melgas datan del siglo XV d.C., mientras que los andenes se corresponderían, de acuerdo con evidencia indirecta, con un periodo posterior, esto es, cuando ingresó el Imperio inkaico a esta zona, aunque todavía es necesario reunir más evidencia para contrastar esta hipótesis preliminar.
Los andenes se presentan segregados sin asociación directa a un poblado particular pero muy cerca de un tramo del Qhapaq Ñan. Su emplazamiento aislado en espacios escabrosos se condice con una estrategia que los inkas empleaban a fin de aminorar el impacto sobre la productividad agrícola local, esto es, construir infraestructura agrícola en áreas que habían sido poco usadas durante el período inmediatamente anterior (Williams 2000: 64).

Los conjuntos de andenes se posicionan sobre laderas y conforman entre 70 y 100 plataformas planas que, en promedio, miden $100 \mathrm{~m}$ de largo por $1,20 \mathrm{~m}$ de alto aproximadamente. Estas estructuras fueron construidas en desnivel con una pendiente promedio del $25 \%$, formadas por muros transversales inclinados unos grados hacia el relleno. Las paredes tienen materiales sedimentarios intersticiales que no serían el resultado de los procesos de deposición eólica, sino de origen antrópico. A diferencia de los despedres encontrados en el sector del río Grande de San Juan Mayo, los registrados en este sector son muy escasos y presentan pequeños amontonamientos de rocas que no llegan a superar los $9 \mathrm{~m}^{2}$. En principio, no se registran procesos continuos de depositación de piedras como producto de la limpieza de las parcelas y tampoco se registran en los sondeos realizados indicios de producción agrícola a largo plazo. ${ }^{5}$

Estas estructuras se destacan en el paisaje $y$, de acuerdo con las prospecciones arqueológicas realizadas al Sur de Pozuelos y en la cuenca de Santa Catalina en los últimos años, no se conocen otras de tal magnitud. Los primeros datos obtenidos revelan la ausencia de procesos de mantenimiento y remodelación de los muros. Los sondeos realizados nos permitieron reconocer que las paredes no están superpuestas, esto es, no se detecta una secuenciación con relaciones de adosamiento como sí fue demostrado en otras estructuras agrícolas del NOA (Quesada y Maloberti 2012). Si bien resulta complejo hacer interpretaciones cronológicas, creemos que es importante destacar que los andenes habrían sido construidos de forma contemporánea.

Entre los andenes se detectaron tres recintos subcuadrangulares de $3 \mathrm{~m} \times 2 \mathrm{~m}$ aproximadamente, dos de los cuales fueron seleccionados para su excavación parcial. El recinto "A" no presentaba restos arqueológicos ni a nivel superficial ni subsuperficial. El recinto "B" presentaba alteraciones por la erosión y por reocupaciones de periodos posteriores. Pese a los procesos posdepositacionales 
observados, se extrajo carbón vegetal del recinto "B", de la unidad estratigráfica 110, a $15 \mathrm{~cm}$ de profundidad. La muestra fue datada en $175 \pm 23$ años AP con dos posibles rangos de edades entre 1662-1693 DC y 1727-1812 DC (calibrado a 2 sigmas con el programa CALIB 2.0 [Stuiver y Reimer 1986]). Presumimos que se trata de una reocupación colonial, como se observa también en otros sectores del sur de Pozuelos. En este sentido, sabemos que en el siglo XVII no hay evidencia de ocupaciones a gran escala para manejar esos andenes, por lo cual es muy factible que no haya sido construido en esa época, sino que se trate de un evento de reocupación.

Entre las melgas identificadas en Carahuasi se reconocieron recintos circulares individuales; uno de ellos fue excavado parcialmente y a $50 \mathrm{~cm}$ de profundidad se extrajo carbón vegetal datado en $519 \pm 28$ AP (unidad estratigráfica 201, a 43 cm de profundidad. Recinto C. UP 1) con dos rangos de edades entre 1423-1446 d.C. y 1409-1453 d.C. (calibrado a 2 sigmas con el programa CALIB 2.0 [Stuiver y Reimer 1986]).

Los análisis preliminares de microfósiles, realizados sobre el material sedimentario ${ }^{6}$ de los andenes, revelan la presencia de granos de almidón individuales de formas circulares y poliédricos de los tipos presentes en maíz (Zea mays) (Korstanje y Babot 2007). También se encontraron almidones que pueden hallarse en Solanum tuberosum L. (papa). En todas las muestras se registraron distintos porcentajes de presencia de gramíneas correspondientes a la subfamilias Panicoideae, Pooideae y Chloroideae.

A diferencia de lo que se observa en el área de San Juan Mayo, Ios despedres en Carahuasi son escasos y de un tamaño considerablemente menor. La muraria presenta escasa selección de materiales, sin canteo u orientación predominante, construida con rocas de mayor tamaño en la base, a partir de las cuales se asientan filas de otras pequeñas y medianas. Las rocas empleadas para la manufactura de estos muros fueron Ilevadas hacia las estribaciones desde otros sectores pero de la misma localidad.

Considerando las dataciones radiocarbónicas realizadas en los dos sectores agrarios de Moreta, sus características arquitectónicas y las técnicas de construcción, podemos plantear que el paisaje agrario es resultado de un proceso diacrónico.

\section{DISCUSIÓN DE LOS PRIMEROS RESULTADOS}

Las poblaciones chicha se destacaron por un gran conocimiento técnico en el uso sistemático del agua, manifestado en el empleo de colectores, represas y melgas. Su producción se sostenía en el cultivo de pseudocereales como quinoa y amaranto, y otros vegetales como papas y maíz. Hasta el momento, la presencia de poroto y zapallo en el registro arqueológico se limita al contexto de consumo, sin poder constatarse su procedencia.

Las laderas bajas y altas de las quebradas fueron cultivadas y tanto el agua de cauces como de lluvia fue aprovechada para la producción. Los campos de cultivo presentan un diseño centrado en optimizar el uso del agua y en extender las superficies cultivables; el desarrollo agrícola es extenso y está separado espacialmente de otras áreas -residenciales, pastoriles, plazas, etcétera.

Los paisajes agrarios caracterizados en este trabajo registran en su fisonomía arqueológica una coyuntura histórica que modificó algunas prácticas agrícolas de las poblaciones chicha. Por un lado, la manifestación de un aumento demográfico excepcional en la microrregión El Angosto durante el siglo XIII (Nielsen et al. 2015: 55), y por otro, en Moreta, un cambio de espacialidad en el siglo XIII y la Ilegada del Inka al asentamiento a mediados del siglo XV. En general, el discurso arqueológico interpreta que estos tipos de eventos afectan directamente la escala de gestión del trabajo, lo que significa un manejo supradoméstico con roles jerárquicos y con objetivos dirigidos a intensificar el volumen de producción. Sin embargo, como discutiremos a continuación, los casos estudiados no se condicen con esta idea generalizada.

Durante los primeros siglos del segundo milenio de la Era, la población del sur de Pozuelos se distribuyó por el territorio en comunidades pequeñas, con una orientación agrícola y/o pastoril a baja escala. Sin embargo, estos diminutos caseríos convivieron con transformaciones políticas regionales que impactaron en el área, como puede verse con la nueva espacialidad constituida en Moreta mediante la construcción de un conglomerado habitacional segregado de campos de cultivo durante los siglos XIII y XIV. Con posterioridad, por el arribo del Inkario a la región, se agregó un recinto perimetral compuesto (RPC), un tramo del Qhapaq Nan, corrales y, aparentemente, andenes. 
Vale aclarar que, en general, entre los siglos $X$ y XV d.C., la producción se mantuvo mayoritariamente a baja escala al sur de Pozuelos, gestionada a nivel doméstico y eventualmente comunitario, como fue demostrado por recientes investigaciones en la zona. En este sentido, se mantuvo cierto grado de autonomía, pese a la aparición de proyectos colectivos en los que había comenzado a operar con fuerza un nivel más supradoméstico de relaciones. Si los andenes son producto de la llegada del Inka, como venimos hipotetizando, podemos plantear que, de acuerdo con las características descriptas más arriba, su explotación se llevó a cabo pero sin la intención de afectar la productividad local doméstica. Esto significa que los grupos de familias no habrían cambiado de forma radical su modo de vida y de producir alimentos.

La secuencia de estructuras agrarias descriptas en este trabajo no se condice con un proceso de intensificación agraria (Albeck 2010). En principio, las recolecciones superficiales y las excavaciones arqueológicas dieron una baja y casi nula presencia de restos (i.e., cerámica, lítico, macrorrestos botánicos, etc.); casi no hay despedres producto de la limpieza y mantenimiento de los campos, las matrices de las estructuras estudiadas presentan características correspondientes a un uso único, sin superposición, derrumbe o remodelación en estratigrafía. No se detectó evidencia de almacenamiento, ni hay indicios arqueobotánicos relacionados con la mejora del rendimiento de los suelos (i.e., esferulitas); tampoco se reconocieron grandes obras de riego ni cierta planificación en las estrategias de cultivo. En este sentido, es posible reconocer que, más que centrarse en la inversión de tiempo y trabajo en la construcción de canales y/o de estructuras de almacenamiento, prefirieron instalar un conjunto de estructuras con gran visibilidad, ${ }^{7}$ en un área sin modificaciones antrópicas hasta ese momento, y a la vista de otras poblaciones. De esta manera, esta arquitectura, más que apuntar a la intensificación en búsqueda de capital exclusivamente económico, se enfocó posiblemente también en la acumulación de otros tipos de capital -simbólico- (Bourdieu 1990) como una forma de control y delimitación territorial. Los andenes de Carahuasi presentan una gran visibilidad al alcance de dos pukaras -Rinconada y Moreta- $y$, desde allí, es posible observar también otros espacios significativos de esa época, como el Abra de Queta, un tramo del Qhapaq Ñan, la laguna de Pozuelos, dos montañas sagradas o apus -cerro Bonete y el volcán Granada-. En consecuencia, puede que estos sitios agrícolas no solo hayan sido espacios de producción, sino también sectores de control de la tierra durante coyunturas complejas con tensiones y movimiento poblacional (Nielsen et al. 2015).

Los datos obtenidos de los sectores residenciales en el San Juan Mayo expresan un crecimiento sustancial durante el siglo XIII d.C.; poblaciones dispersas pasaron a ser poblados densos constituidos por viviendas aglomeradas sin asociación directa a campos de cultivo (Nielsen et al. 2015). En ese sentido, sospechamos que los espacios de producción de estas nuevas ocupaciones se encuentran en estas quebradas aisladas no solo porque no hay otros asentamientos para discutir potenciales artífices, sino porque además se expresan como un gesto sincrónico, un proyecto colectivo construido de manera planificada, a juzgar por la homogeneidad de la arquitectura (i.e., regularidad en los trazados, interdependencia de estructuras, canales que integran varios sitios). Estas obras se extienden por kilómetros, lo que implicaría una administración coordinada aunque no necesariamente centralizada, y esto porque no se registran indicios de centralidad en el almacenaje, ni presencia de plazas con espacialidad jerarquizada, acceso diferencial a recursos, etc. La homogeneidad material nos impide pensar en relaciones supradomésticas centralizadas (Nielsen et al. 2015). De esta forma, la incorporación de este proyecto agrícola colectivo podría concebirse como una táctica ${ }^{8}$ (De Certeau 1999) para lidiar con un mayor índice de población y transformar la situación de manera beneficiosa para la comunidad en el momento preciso. Si bien no podemos establecer una medida exacta, sabemos que la autonomía doméstica se vio atravesada y perdió peso en comparación con periodos precedentes, y que muchas decisiones recayeron en el ámbito comunitario.

Al igual que otras poblaciones de la zona, los asentamientos chicha se vieron afectados sobre todo entre los siglos XIII y XVI d.C. por las transformaciones sociales sufridas en los Andes circumpuneños con estados de guerra, sequías y redistribución poblacional (Nielsen et al. 2015). En ambas áreas estudiadas se reconoció que ciertas estructuras de cultivo (i.e., quebrada de Saratarkuna, quebrada Pabellón, quebrada de San Lorenzo, Moreta 1 y Carahuasi) fueron parte de un entramado complejo conformado por coyunturas políticas regionales. En definitiva, como primera conjetura sobre la base de las investigaciones realizadas hasta ahora, 
planteamos que los espacios de producción respondieron, posibilitaron y condicionaron las estrategias empleadas por las poblaciones frente a los cambios. En San Juan Mayo, durante el período de Desarrollos Regionales, los sistemas de cultivo se posicionaron como una táctica para lidiar con problemas de abastecimiento y crecimiento demográfico (Nielsen et al. 2015), mientras que en Moreta fueron agregándose nuevas formas de cultivar al compás de los cambios que se sucedían durante los períodos tardíos e inka.

\section{Agradecimientos}

Las investigaciones fueron financiadas mediante subsidios PICT (FONCYT), PIP (CONICET) y PIUNT (Universidad Nacional de Tucumán). Agradecemos muy especialmente a cada uno de los habitantes del sur de Pozuelos por permitirnos trabajar en su territorio por más de una década y al Dr. Axel E. Nielsen por su dirección en las investigaciones realizadas en el San Juan Mayo.

\section{REFERENCIAS CITADAS}

Acuto, F. A.

2007 Fragmentación vs. Integración comunal: repensando el período tardío del noroeste argentino. Estudios Atacameños 34: 71-95.

Albeck, M. E.

1993 Contribución al estudio de los sistemas agrícolas prehispánicos de Casabindo (Puna de Jujuy). Tesis Doctoral inédita. Universidad Nacional de la Plata, Facultad de Ciencias Naturales y Museo, La Plata. 2010 Estudios de agricultura prehispánica: Casabindo (1980-1993). En Arqueología de la agricultura. Casos de Estudio en la región andina argentina, editado por M. A. Korstanje y M. Quesada, pp. 12-47. Magna, San Miguel de Tucumán.

Angiorama, C., M. J. Pérez Pieroni y M. F. Becerra 2017 Moreta, "pueblo de yndios chichas y tambo del yunga" (Puna de Jujuy, Argentina). Estudios Atacameños 55: 163-181.

Bourdieu, P. 1990 Espacio social y génesis de las clases En Sociología y cultura, pp. 281-310. Grijalbo, México.

Carandini, A. 1997. Historias en la Tierra: manual de excavación arqueológica. Crítica, Barcelona.
De Certeau, M.

1999 La invención de lo cotidiano: artes de Hacer. Iberoamericana, México.

Díaz, R. A.

2009 Historias de tierra y agua: Introducción a los espacios agrícolas prehispánicos de Laguna Blanca. El caso de estudio de la Aldea Arqueológica Piedra Negra (Dpto. Belén, Provincia de Catamarca). Tesis de Licenciatura inédita. Escuela de Arqueología, Universidad Nacional de Catamarca, Catamarca.

Figueroa, G.

2008 Los sistemas agrícolas del Valle de Ambato, Catamarca, siglos VI a XI d.C. Intersecciones en Antropología 9: 313-317.

Franco Salvi, V.

2012 Estructuración social y producción agrícola prehispánica durante el primer milenio d.C. en el valle de Tafí (Tucumán, Argentina). Trabajo Final para optar al grado de Doctor en Historia. Universidad Nacional de Córdoba, Córdoba.

Harris, E. C.

1991. Principios de Estratigrafía Arqueológica. Traducido por E. Junyent. Crítica, Barcelona.

Korstanje, M. A. y M. del P. Babot

2007 A microfossil characterization from South Andean economic plants. En Places, people and plants: using phytoliths in Archaeology and Palaeoecology, editado por M. Madella, M. K. Jones y D. Zurro, pp. 41-72. Oxbow Books, Cambridge.

Krapovickas, P.

1983 Las poblaciones indígenas históricas del sector oriental de la Puna (un intento de correlación entre la información arqueológica y la etnográfica). Relaciones de la Sociedad Argentina de Antropología 15: 7-24.

Nielsen, A. E.

2006 Pobres Jefes: Aspectos corporativos en las formaciones sociales pre-inkaicas de las Andes Circumpuneños. En Contra la tiranía tipológica en arqueología: Una visión desde Sudamérica, editado por C. Gnecco y C. H. Langeback. pp. 121-150. UniandesCESO, Universidad de los Andes, Bogotá.

Nielsen, A. E., C. I. Angiorama, J. Maryañski, L. López y F. Ávila

2015. Paisajes prehispánicos Tardíos en San Juan Mayo (frontera Argentina - Bolivia). Arqueología 21: 29-61.

Olivera, D.

2001 Sociedades agropastoriles tempranas: el formativo inferior del noroeste argentino. En Historia Argentina prehispánica, t. I, editado por E. Berberián y A. Nielsen, pp. 83-125. Brujas, Córdoba. 
Quesada, M.

2006 El diseño de las redes de riego y las escalas sociales de la producción agrícola en el $1^{\circ}$ Milenio DC (Tebenquiche Chico, Puna de Atacama). Estudios Atacameños 13: 31-46.

Quesada, M y A. Korstanje

2010 Cruzando estructuras: el espacio productivo y su entorno percibido desde las prácticas cotidianas. En El hábitat prehispánico, editado por M. E. Albeck, M. C. Scattolin y M. A. Korstanje, pp. 123-153. Facultad de Humanidades y Ciencias Sociales, Universidad Nacional de Jujuy, Argentina.

Quesada, M. y M. Maloberti

2012 La expansión de los espacios agrícolas. Continuidades en la construcción del paisaje agrario en el sector septentrional del Valle de El Bolsón (siglos I a XV). En Racionalidades campesinas en los Andes: pasado, presente, editado por P. Cruz., R. Joffre y T. Winkel, pp. 140-165. Institut de Recherche pour le Développement, Montpellier.

Raffino, R. A.

1988 Poblaciones indígenas en Argentina. Urbanismo y proceso social precolombino. Ediciones TEA, Buenos Aires.

Sempé, M. C.

2005 El período Tardío en Azampay: El señorío Belén y su modelo geopolítico. En Azampay Presente y Pasado de un pueblito catamarqueño, pp. 365-380. Al Margen, La Plata.

Stuiver, M. y Reimer, P. J.

1986 A computer program for radiocarbon age calibration. Radiocarbon 28 (2B): 1022-1030.

Williams, $\mathrm{V}$.

$2000 \mathrm{El}$ imperio Inka en la provincia de Catamarca. Intersecciones en Antropología 1 (01): 55-78.

Williams, V., A. Korstanje, P. Cuenya y M. P. Villegas 2010 La dimensión social de la producción agrícola en un sector del Valle Calchaquí medio. En Arqueología de la agricultura, editado por A. Korstanje y M. Quesada, pp. 178-207. Magna, San Miguel de Tucumán.

\section{NOTAS}

1. Las investigaciones realizadas por Nielsen et al. (2015) indican una expansión rápida del asentamiento principal de la quebrada, que durante la primera mitad del siglo XIII pasó de ser un área residencial dispersa y a baja escala a cubrir un total de 5,5 ha densamente edificadas. Este fenómeno no fue fruto del crecimiento demográfico local, sino que habría implicado procesos de redistribución poblacional.
2. Para regar la superficie de esta quebrada se requirió de la construcción de un canal principal y otros secundarios junto con una estructura que maximizara el aprovechamiento del agua, esto es, la "melga", pero para cuya edificación, a su vez, se necesita un terreno limpio, lo que implica también el uso de palas y/o azadas (en las superficies de cultivo no se hallaron, pero sí se encontraron numerosos implementos en las viviendas de Finispatriae, el asentamiento principal del área). La presencia de melgas en las quebradas implica directamente la formación de despedres, como así también la presencia de canales y de implementos agrícolas. Este tipo de estructura se construye durante los meses fríos, por tratarse de un periodo sin crecimiento vegetativo de los cultivos y con suelos secos-porosos que facilitan la ejecución y planificación de la construcción.

3. En un recinto circular identificado entre el conjunto de estructuras agrícolas se realizó un sondeo, lo que nos permitió obtener una muestra de carbón vegetal para AMS (unidad estratigráfica 100, a $43 \mathrm{~cm}$ de profundidad. Sondeo 1, en Estructura 1), que arrojó un fechado de $675 \pm$ 45 y dos posibles rangos de edades de 1260-1330 d.C. y 1338-1398 d.C. (calibrado a 2 sigma con el programa CALIB 2.0 [Stuiver y Reimer 1986]). Por otra parte, se extrajo carbón vegetal de un recinto denominado "Recinto Extremo Oeste" (unidad estratigráfica 501, a $70 \mathrm{~cm}$ de profundidad. Sondeo 1), con una datación de $559 \pm 28$ años, con dos posibles rangos de edades entre 1310-1360 d.C y 1386 d.C. y 1428 d.C. (calibrados a 2 sigmas con el programa CALIB 2.0 [Stuiver y Reimer 1986]).

4. En el asentamiento de Moreta, a partir del siglo XIV se registra una espacialidad diferente, donde las unidades residenciales se aglutinan y están segregadas de los campos de cultivo (Angiorama et al. 2017). Moreta 1 y las melgas de Carahuasi fueron datadas para este período $y$, en principio, es posible plantear, por su contemporaneidad y asociación espacial, que fueron las parcelas que explotaban estas poblaciones de forma conjunta.

5. En un sector de canal del sitio se realizó un sondeo de $1 \mathrm{~m} \times 1 \mathrm{~m}$ y $0,33 \mathrm{~m}$ de profundidad -llegando al estrato estéril-. La matriz de Harris registra la presencia de cuatro unidades estratigráficas, una de las cuales (UE 123) corresponde a un rasgo de canal. A diferencia de otros canales excavados en sitios arqueológicos multicomponente (Franco Salvi 2012), no se registraron lentes superpuestos y la presencia de materiales arqueológicos fue nula. En ese sentido, interpretamos que el manejo de este conjunto agrícola se habría dado en un plazo único, no diacrónico, aunque no podemos inferir duración y cronología. Los sondeos realizados en las parcelas de los andenes revelan un solo depósito arqueológico, y no se observa la presencia de una capa arqueológica que devele procesos de remodelación o mantenimiento, rasgos, derrumbes, etc. Si comparamos estos sondeos con los registrados en estructuras de cultivo explotadas a largo plazo (Franco Salvi 2012), es posible plantear que fue utilizado en un único periodo y muy probablemente durante poco tiempo.

6. Se realizaron cuatro sondeos de $1 \times 1 \mathrm{~m}$ en las parcelas asociadas a las paredes de los andenes y se extrajeron ocho muestras de suelo a nivel vertical de cada sondeo, de abajo arriba y en zigzag. Los perfiles a muestrear se eligieron mediante muestreo aleatorio y se tuvieron en cuenta los recaudos necesarios para evitar su contaminación. Asimismo, se obtuvieron muestras inalteradas como "muestras testigo" para efectuar la comparación con el 
sedimento arqueológico. En cada una de ellas se observaron un mínimo de 200 silicofitolitos y 300 microfósiles. La identificación de los fitolitos se realizó mediante una clave elaborada con base en los estudios de material comparativo de la vegetación de la región y la información bibliográfica referente al tema.

7. El grado de visibilidad se midió mediante software aplicado, en este caso particular, la herramienta View Shed Tool de global Mapper.
8. Una población pone la táctica en acción a partir de la imposición de una fuerza externa, esta se presenta audaz y da importancia no al lugar sino al tiempo, actúa en el momento preciso y transforma la situación de manera beneficiosa para ellos. 